\title{
Stochastic Dominance : An Application to the Insurance Portfolio Decision
}

\author{
by Devinder K. Gandhi *, Anthony Saunders ** and Edmund G. Sugars ***
}

\section{Introduction}

Periodically, insurance company managers must make decisions governing the mix of policies to be underwritten, the scale of the underwriting operation, the extent of regional diversification, the types and amounts of reinsurance, and the manner in which cash is to be invested. The number of competing decisions in each of these decision classes being large, the total number of sets of decisions tends to be very large. Each set would generate a separate insurance and investment portfolio; a corporate portfolio. The financial outcomes for each corporate portfolio can be represented by a probability distribution or by a cumulative probability distribution of policyowners' surplus at a defined future date.

Were this very large number of distributions specified (a formidable managerial problem in itself), established methods for identifying the probability of ruin for each corporate portfolio could be employed and those distributions that indicate intolerably high probabilities of ruin eliminated from further consideration. ${ }^{1}$ The manager would then have a smaller but still difficult problem; that of deciding which remaining distribution, and thus, set of decisions, is optimal for the company. Optimum choice demands specification of optimization criteria.

This paper addresses the problem of specifying appropriate criteria. It will be argued that Stochastic Dominance (SD) criteria are superior or more efficient, in many cases, than those derived from traditional models. The case in favour of SD rests on three points :

(a) SD rules rest upon the axioms of rational choice underlying utility theory ;

* Associate Professor of Finance, Concordia University, Canada.

** Assistant Professor of Finance, Graduate School of Business, New York University.

*** Professor of Management, University of Calgary, Canada.

1 Ruin models have been discussed by Sugars [11] and Wooddy [13] 
(b) SD rules incorporate all of the information in each prospective wealth distribution, rather than just the information encapsulated in the first two or three moments of the distribution $;^{2}$ and

(c) SD requires relatively undemanding assumptions as to the specific form of the utility function used by the decision maker.

This paper first discusses the three basic SD rules and gives some diagrammatic examples. $^{3}$ It then compares SD with established models of choice. A simple insurance example is then discussed. The final part discusses the possibilities and problems in the practical application of SD rules.

\section{Stochastic dominance decision rules}

SD is a choice-theoretic procedure for preference ordering of uncertain prospects. In the insurance case, SD facilitates preference ordering for alternative corporate portfolios. The three SD rules (First Order, Second Order and Third Order) coincide with progressively more restrictive assumptions concerning the decision maker's utility function. The First Order rule represents the strongest ordering conditions because it places the fewest restrictions on the utility function. However, the set of distributions orderable under the First Order rule is generally smaller than the set orderable under the other two rules. That is, a pair of distributions orderable under Third Order rule will be orderable under the remaining sets of rules but the reverse may not be true.

Each of the three rules is now examined in detail.

\subsection{First-Order Stochastic Dominance (FSD)}

This rule applies when it is safe to assume that the governing utility function is a non-decreasing function of increasing wealth ; more precisely, that the first derivatives at every point within the relevant range of the uncertain wealth outcomes are continuous and never smaller than zero. This class of utility functions has wide applicability since it includes all risk-seeking, risk-averting and risk-indifferent individuals who never prefer less wealth to more wealth. ${ }^{4}$

2 The mean-variance model is demonstrably inferior to a mean-variance-skewness model. For example, an insurer manager faced with the task of choosing one of two loss distributions with equal means and variances would presumably employ differences in skewness of the distributions to make his choice. By similar logic, a four moment model is superior to a three moment model and an $n$ moment model is superior to an $n-1$ moment model.

3 Proofs of these rules are given by Hadar and Russell [5], Hanoch and Levy [7], Quirk and Saposnik [10] and by Whitmore [12] but will not be given in this paper.

4 It also includes individuals who comply with the Friedman and Savage [4] hypothesis that a given individual may be risk-averse over some ranges of prospective wealth and riskseeking over others. FSD rules were originally developed by Quirk and Saposnik [10] who applied the term "Stochastic Dominance" to FSD. Hadar and Russell [5] provided the "First Order" qualifying term. 
Figures 1 (a) and 1(b) show probability and cumulative probability distributions (CPD) of terminal wealth, $w$, for two hypothetical corporate portfolios $A$ and $B .^{5}$

The FSD rule specifies that $B$ be chosen provided that :

(1) the governing utility function meets the criteria discussed above ; and

(2) $B^{\prime} \leqslant A^{\prime}$ for every point within the domain of $w$; and

Figures $1(a)$ and $1(b)-W e a l t h(w)$ distributions for $A$ and $B$.

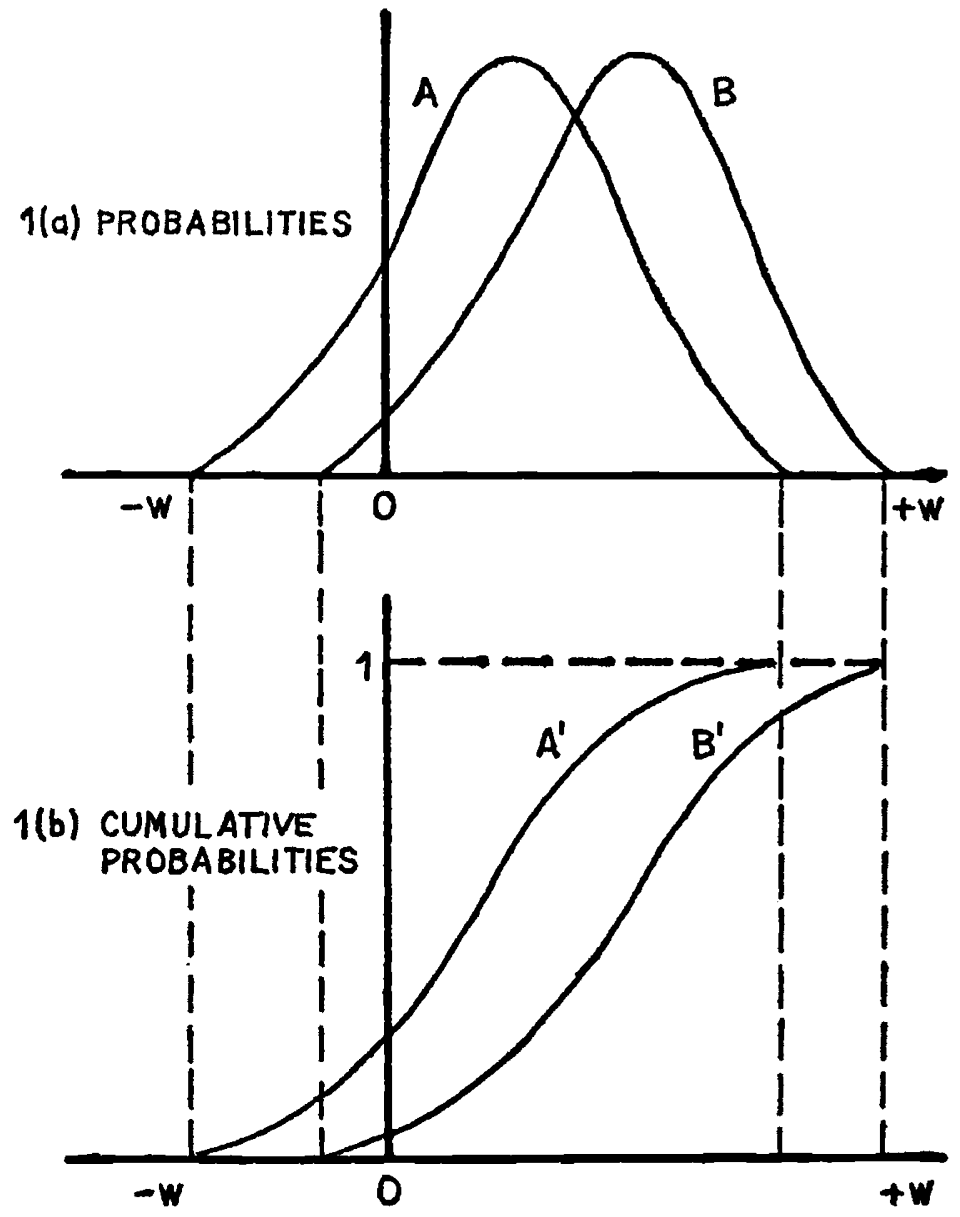

5 Terminal wealth, $w$, is a random variable representing the values of policyowners' surplus at some specified future date. 
(3) for at least one value of $w, B^{\prime}<A^{\prime} .^{6}$

Thus, in this example, the CPD for $B, B^{\prime}$, dominates that for $A, A^{\prime}$; for every value of $w$ the probability of $w$ or more occurring is not smaller with $B$ than it is with $A$. Visually, the entire graph of $B^{\prime}$ lies to the right of $A^{\prime} .^{7}$

\subsection{Second-Order Stochastic Dominance (SSD)}

The preceding case is clearcut. However, the FSD rule would be violated were $A^{\prime}$ and $B^{\prime}$ simply-intertwined as shown in Figure $2(\mathrm{~b})$ or, indeed, were $A^{\prime}$ and $B^{\prime}$ to cross more than once. However, the SSD rule may permit a clear-cut choice provided that the manager assumes that the governing utility function takes a less general form than that applicable to FSD.

The SSD rule applies only when marginal utility is not an increasing function of terminal wealth. Mathematically, the second derivatives of the utility function are never greater than zero. Thus, the utility functions to which SSD applies may reflect riskaversion and/or risk-indifference, but unlike those for FSD, never reflect risk-seeking.

Figures 2(a), 2(b) and 2(c) show probability distributions, cumulative probability distributions and the integrals of the cumulative probability distributions over the domain of $w$, respectively, for hypothetical corporate portfolios $A$ and $B$. The SSD rules require that $B$ be chosen provided that :

(1) the governing utility function meets the criteria applicable to SSD ; and

(2) $B^{\prime \prime} \leqslant A^{\prime \prime}$ for every point within the domain of $w ; 8$ and

(3) for at least one value of $w, B^{\prime \prime}<A^{\prime \prime}$.

Thus, in Figure 2(c), $B^{\prime \prime}$ dominates $A^{\prime \prime}$ for every value of $w$; the entire graph of $B^{\prime \prime}$ lies to the right of that for $A^{\prime \prime} .^{9}$ identical.

6 This condition is necessary to eliminate the degenerate case where the CPDs are

7 In the FSD case it can be readily shown that the mean of the dominant CPD will always exceed that of the dominated. However, a similarly consistent relationship between the variances of the two distributions cannot be specified. Furthermore, the probability of ruin in the dominant CPD will always be less than that of the dominated.

8 More precisely, $A$ is not preferred to $B$ if, and only if, $\int_{a}^{w}(A(y)-B(y)) d y \geqslant 0$ for each value of $w$, where $a$ is the lowest point on the range of either $A$ or $B$.

9 In the simply-intertwined case, the CPD's intersect but once; in this special case the necessary and sufficient conditions for $B$ to dominate $A$ are that (a) the mean of $B$ equals or exceeds that of $A$ and (b) the lowest probable value of $w$ for $A$ is below that for $B$ and the probability associated with the lowest value of $A$ is higher than that of the lowest value of $B$. Hammond [6] shows that this special case occurs when both distributions being compared belong to one of several families of distributions (for example the normal distribution family, the gamma distribution family, etc.). 
Figures $2(a), 2(b)$ and $2(c)$-Wealth (w) distributions for $A$ and $B$.

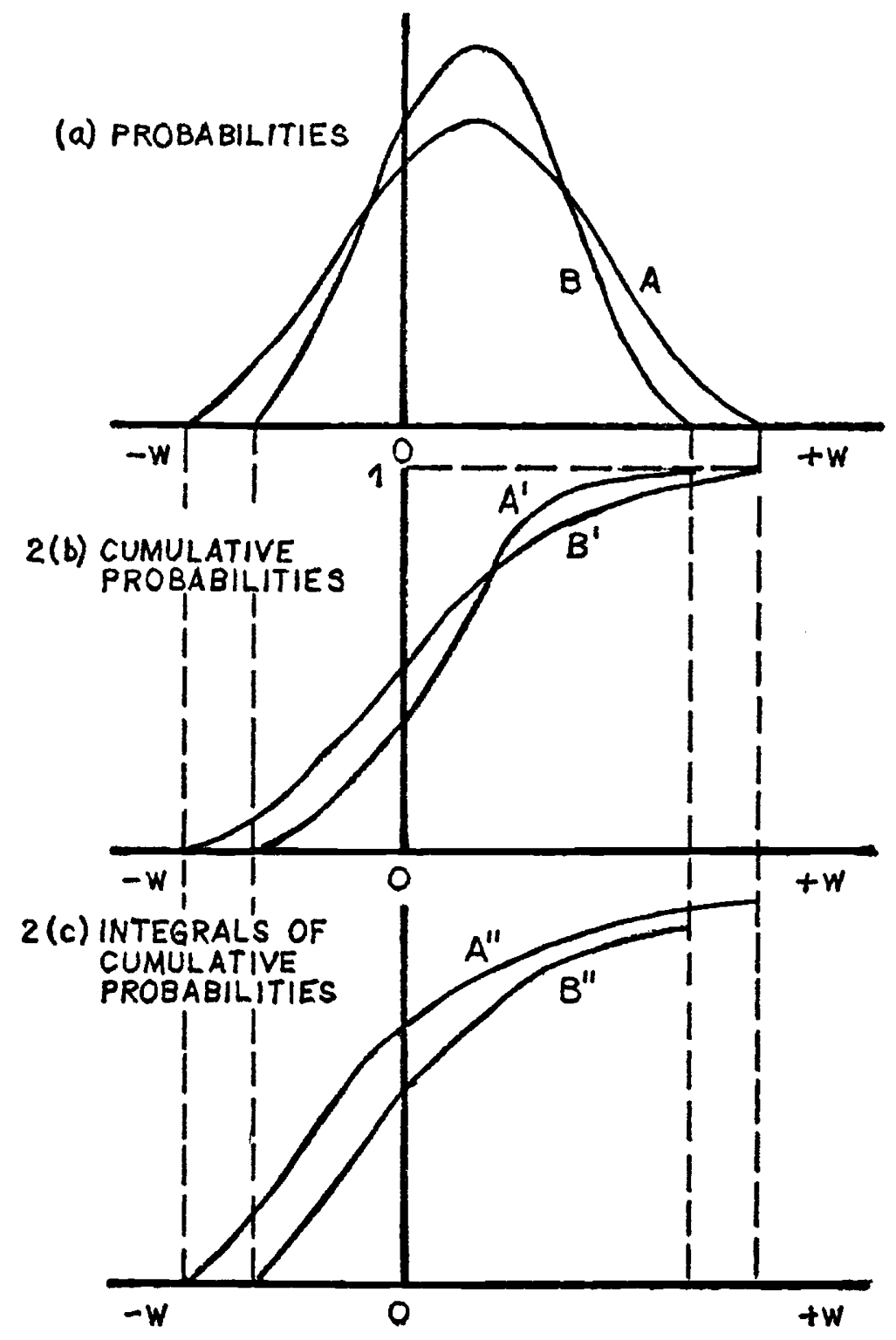




\subsection{Third-Order Stochastic Dominance (TSD)}

In complex cases $A^{\prime \prime}$ and $B^{\prime \prime}$ may also intersect, which precludes the application of FSD and SSD rules. However, the TSD rule, developed by Whitmore [12], may provide a solution provided that a more restrictive utility function assumption than that made for either FSD or SSD is accepted.

TSD applies when, in addition to the utility function restrictions required by FSD and SSD, the utility function is restricted to one for which all third derivatives equal or exceed zero. ${ }^{10}$

TSD requires that corporate portfolio $B$ be chosen over $A$ provided that :

(1) the governing utility function meets the criteria immediately preceding ; and

(2) $B^{\prime \prime \prime} \leqslant A^{\prime \prime \prime}$ for every $w$, where $\mathrm{B}^{\prime \prime \prime}$ and $A^{\prime \prime \prime}$ represent the double integrals of the CPDs for $B$ and $A$, respectively ; and

(3) for at least one value of $w, B^{\prime \prime \prime}<A^{\prime \prime \prime}$; and

(4) $B^{\prime \prime}<A^{\prime \prime}$ for the largest value in the domain of $w .11$

\section{An evaluation of SD rules}

The comparative strengths and weaknesses of SD rules demand closer scrutiny. The basic components of the established decision models that compete with SD are the expected value of future wealth, the variance of future wealth and the decision makers' precise utility function. These components have been combined to produce the mean-variance (MV), coefficient of variation (CV) and expected utility (EU) models. Exhaustive comparisons of SD with each of the preceding, at the theoretical, empirical and numerical levels, would be too lengthy. Accordingly, only a brief qualitative critique of the basic components of the models noted will be given, followed by a simple numerical example.

\subsection{Qualitative comparison}

Were expected values of competing probability distributions of future wealth used as the sole criterion for choice, at least one of the following assumptions is implied :

10 The natural logarithm utility function falls into this class. Despite its relative restrictiveness, this form appears to closely reflect the preferences of the majority of decision makers. Arrow [2] empirically argues that greater wealth leads to a greater propensity for the decisionmaker to absorb risk. In the above case the wealthier decisionmaker would not be prepared to pay as high an insurance premium as his less wealthy counterpart.

11 More precisely, $A$ is not preferred to $B$ if the integral over the entire domain of $w$ does not meet the conditions given in footnote 8 . 
(1) that the distributions are identical except for the values of the mean ;

(2) that choices are uninfluenced by other moments of the distributions ;

(3) that decision makers are risk-neutral.

Available evidence suggests that these assumptions are rarely valid.

Were the variances used as the sole criterion similar tenuous assumptions are again implied.

Simultaneous evaluation of the means and variances (MV and CV models) merely serves to lend credence to the assumption that the third and higher order moments are irrelevant to the decision maker. Furthermore, MV may produce ambiguous results, as illustrated by the case where distributions $A$ and $B$ have means of 110 and 108 and variances of 100 and 81 , respectively. Here $A$ has the better mean while $B$ has the better variance ; hence, no choice is possible using MV criteria. The CV model specifies that $B$ should be chosen, since its coefficient of variation of .0833 is smaller than that of $A, .091$. Nevertheless, $\mathrm{CV}$ is not derived from the axioms of rational choice underlying utility theory and is, indeed, only a numerical method for adjusting for scale effects. Thus, there is no assurance that $\mathrm{CV}$ will consistently yield decisions that comply with utility theory.

Were it possible to precisely define the utility function that should govern a decision, the preceding difficulties could be avoided by employing the EU model. However, almost intractable difficulties are associated with measuring individual, let alone, collective utility functions..$^{12}$ Furthermore, the controversy over whose individual or collective utility function should govern the decision remains unresolved. ${ }^{13}$

By contrast, SD employs all information, thus all moments, of the probability distributions of future wealth and rests upon the axioms of rational choice (utility theory). Thus, the previous assumptions need not be made and the problems with utility functions avoided if the manager is prepared to make relatively undemanding utility function assumptions.

\subsection{A mean-variance example}

The insurance manager must choose one of two very simple corporate portfolios $A$ and $B$. The random values of prospective policy owners' surplus are rectangularly distributed as shown in Figure 3(a). Figure 3(b) shows the corresponding CPD's. From the FSD rule the CPD for $B$ should be chosen.

12 For example, for all common shareholders.

13 Should it be the manager's, the shareholders' or policyowners'? 


\section{Figures $3(a)$ and $3(b)-W e a l t h(w)$ distributions \\ for $A$ and $B$.}

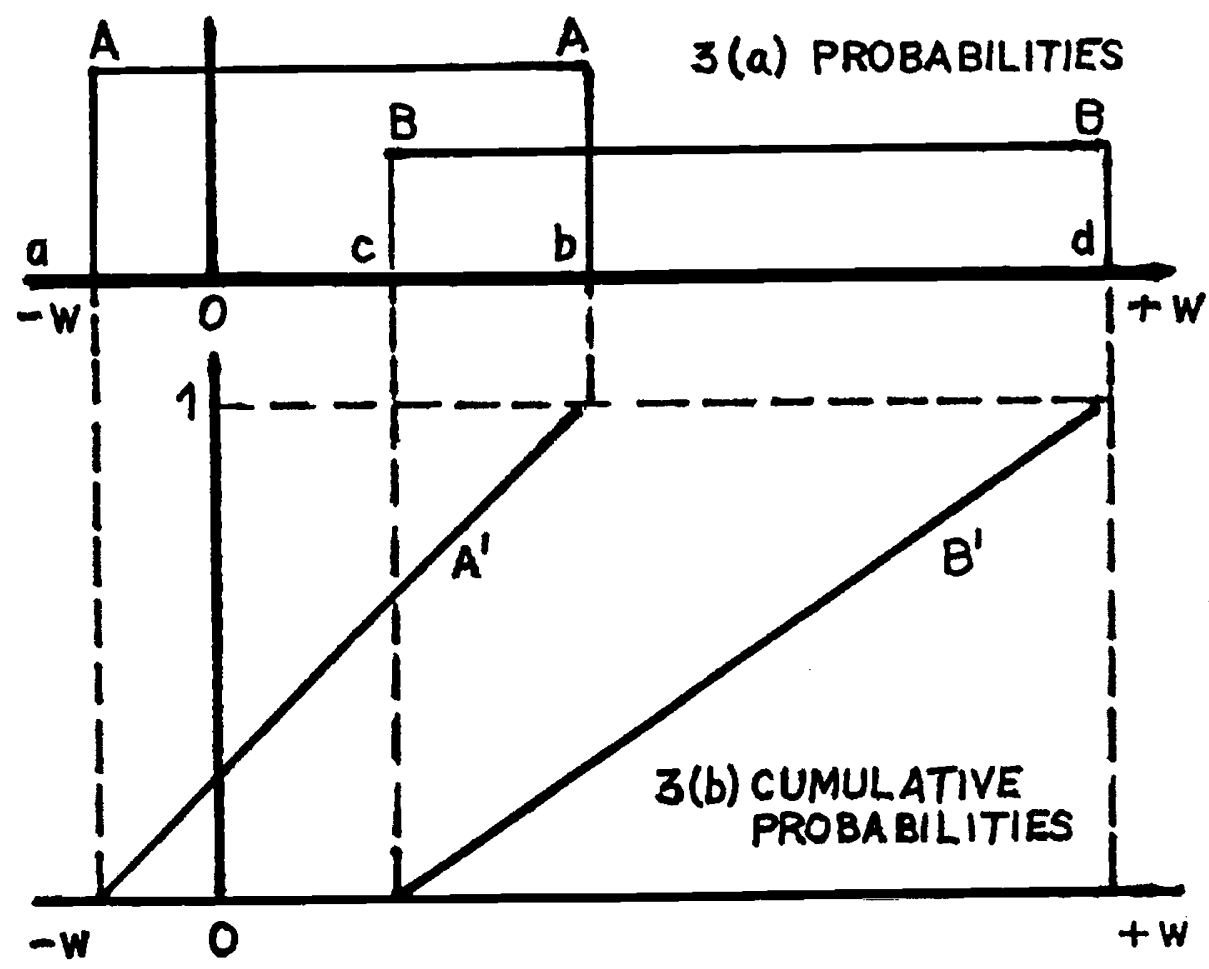

Returning to Figure 3(a), both the mean and the variance of $B$ exceed those of $A$. Thus, expected value prescribes the choice of $B$, while variance prescribes $A$. Indeed, in the absence of further information about the decision maker's risk preferences, $M V$ offers no clear choice. ${ }^{14}$ Furthermore, the CV model may yield choices that either disagree or agree with the SD result. For example, if the dollar values of $a, b, c$ and $d$ shown in Figure 3(a) were set at 4, 6, 5 and 8 respectively. The $C V$ model prescribes the choices of $A$, which is opposite to the SD conclusion.

On the other hand, were the values of $c$ and $d$ set at 5 and 7 , respectively, the coefficients of variation for $A$ and $B$ would equal .115 and .094 , respectively. Thus, $B$ would be chosen which agrees with the SD conclusion.

14 Although there are exceptions (notably in the case of the normal distribution), larger means generally correlate with higher variances, which is the case with rectangular distributions. Hence, MV very often yields no clear choice. 


\section{An Insurance application}

All potential applications of SD to insurance problems and all hypotheses relating SD to insurance cannot be examined here. However, the single example chosen suggests that SD is directly applicable to a wide variety of insurance management decisions.

In this example the manager of a stock company must choose between two corporate portfolios, $A$ and $B .{ }^{15} A$ and $B$ are identical except that the number of policies underwritten in $B$ is twice that of $A$ and half of each policy in $B$ is ceded under a quota share treaty while none of the $A$ policies are reinsured.16

Figures 4(a) and 4(b) show the probability distributions and corresponding cumulative probability distributions of the value of policy owners' surplus for each portfolio on a defined future date. The distances $O P_{0}$ and $O P_{1}$ represent the actual and expected value of policy owners' surplus at the beginning and end of the future time period, respectively. $P_{0} P_{1}$ equals the mean expected increment to $O P_{0}$ during this time period. Ruin occurs if terminal policy owners' surplus drops into the shaded area of the diagram ; the probability of ruin is designated by the shaded negative tails in Figure 4(a). $A$ has a larger tail and, therefore, a higher probability of ruin. However, the manager does not exclude $A$ from further consideration since the probability of defaulting on policy owners' claims is not intolerably high.

The diagram shows that the means of $A$ and $B$ are equal but $B$ has smaller variance. By the Mean-Variance rule $B$ would be chosen. However, neither probability distribution is symmetrical and it is necessary to have utility function information before the choice of $B$ can safely be made.

In the absence of precise utility function information the CPD's are analyzed for stochastic dominance. FSD rules cannot be used because the CPD's cross. However, the manager's calculations show that the integrals of $B$ dominate those of $A$. Hence, $B$ should be chosen if the manager accepts the rather safe assumption that the applicable utility function complies with SSD requirements; that is, it does not reflect riskseeking. ${ }^{17}$

15 A specific managerial problem situation will be used to exemplify the application of Stochastic Dominance Analysis to insurance portfolio decisions. However, many other managerial situations might have been used. Consider, for example, Doherty's article [3] which identifies the conditions, based on Stochastic Dominance Analysis, under which a prospective policy-owner can be induced to accept one risk-sharing mechanism (e.g., deductible) versus another. From the viewpoint of the insurance portfolio manager, Doherty's findings have clear managerial implications. in $B$.

16 Note that the proportions of each class of policy in portfolio $A$ equal the proportions

17 This case is again one of simple intertwining; the special rule given in footnote 9 applies, resulting in the choice of $B$. 
Figures $4(a)$ and $4(b)$ - Policy owner distributions for
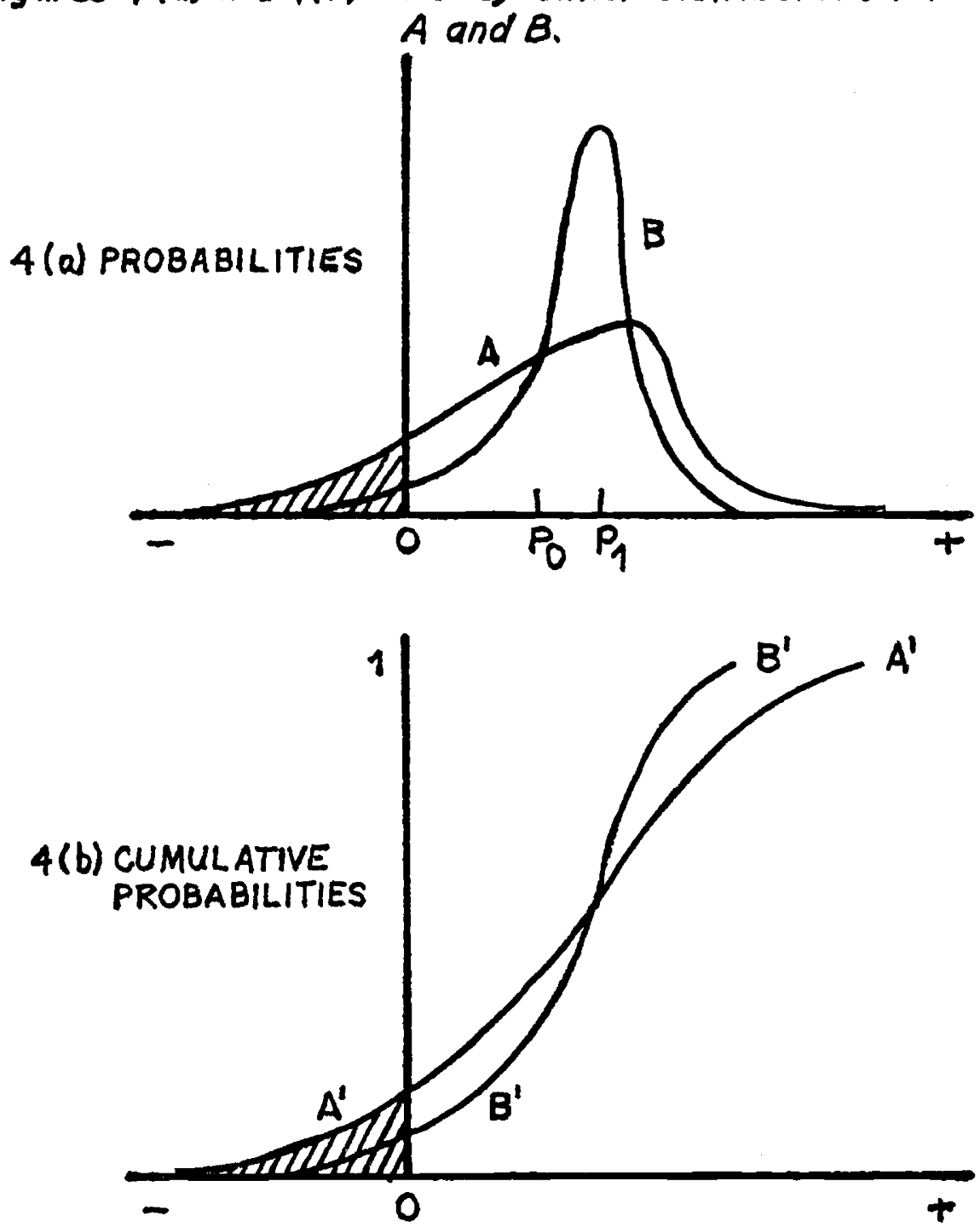

\section{Practical considerations and conclusions}

From the above examples it appears that SD is a conceptually superior approach to decision making under conditions of risk. If this is accepted, the real question is how can SD rules be applied in practice. Indeed, it has to be admitted that SD analysis imposes a slightly greater computational burden on the analyst than $\mathrm{MV}$, 
since (in general) more data points on returns are required to determine accurate and reliable dominance relationships.

The main approach followed so far, in empirical tests of SD, has been to use Monte-Carlo and other simulation techniques to derive CPD's and to compare SD with MV.18

Clearly, the practical use of SD will also depend on the availability of a high speed computer. If one is available, the relevant decision is whether the costs (in terms of computing time and money) are worth the gains in efficiency from using SD instead of MV. A recent paper by Aharony and Loeb [1] assessing the costs and benefits of SD and $\mathrm{MV}$ has shown that the benefits of using SD in investment selection may well outweigh the costs, because of the type and large size of errors produced by using MV criteria.

Thus, SD provides both promising conceptual and practical criteria for insurance management decisions. In practice it is more efficient than MV, largely eliminates the quest for precise measurement of utility functions and sidesteps the unsettled controversy over whose utility functions should govern managerial decisions. Finally, SD complements traditional probability of ruin models by providing criteria for rational choice from those corporate portfolios that remain following elimination of those with intolerably high probabilities of ruin.

18 Porter, Wart, and Ferguson [9] have developed an efficient algorithm DOMIN 2 which can be employed by an analyst to make SD rankings; this simulation model has already been successfully employed in a number of empirical studies. For an empirical comparison of SD and MV, see Porter [8]. 


\section{REFERENCES}

1. AHARONY, J., and LOEB, M.: "Mean-variance vs. stochastic dominance: some empirical findings on efficient sets," Journal of Banking and Finance, 1 (June 1977), 95-102.

2. ARROW, K. J.: Essays in the Theory of Risk Bearing, Markham Co., Chicago, 1971.

3. DOHERTY, N. A.: "Stochastic choice in insurance and risk sharing," Journal of Finance, 32 (June 1977), 921-926.

4. FRIEDMAN, M., and SAVAGE, L. J.: "The utility analysis of choices involving risk," Journal of Political Economy, 56 (August 1948), 279-304.

5. HADAR, J., and RUSSELL, W.: "Rules of ordering uncertain prospects," American Economic Review, 59 (March 1969), 25-34.

6. HAMMOND, J.: "Simplifying the choice between uncertain prospects where preference is non-linear," Management Science, 20 (March 1974), 1047-1072.

7. HANOCH, G., and LEVY, H.: "The efficiency analysis of choices involving risk," Review of Economic Studies, 36 (July 1969), 335-346.

8. PORTER, R. B. : “An empirical comparison of stochastic dominance and mean-variance portfolio choice criteria," Journal of Financial and Quantitative Analysis, 8 (September 1973), 587-608.

9. PORTER, R. B., WART, J., and FERGUSON, D. : "Efficient algorithms for conducting stochastic dominance tests on large numbers of portfolios," Journal of Financial and Quantitative Analysis, 8 (January 1973), 71-82.

10. QUIRK, J.P., and SAPOSNIK, R.: “Admissibility and measurable utility functions," Review of Economic Studies, 29 (1962), 140-146.

11. SUGARS, E. G.: "A managerial theory for the non-life stock company," Journal of Risk and Insurance, 39 (June 1972), 245-256.

12. WHITMORE, G. A.: "Third degree stochastic dominance," American Economic Review, 60 (June 1970), 457-459.

13. WOODDY, J.C.: Risk Theory, a note prepared for the Society of Actuaries, Chicago, 1966. 